\title{
CHARACTERIZATION OF FOULSER'S $\lambda$-SYSTEMS
}

\author{
M. L. NARAYANA RAO
}

1. Introduction. In 1967 D. A. Foulser [1] defined a class of finite Veblen Wedderburn systems called $\lambda$-systems. These systems include regular nearfields and André systems. However there are other finite Veblen Wedderburn (VW) systems which are not $\lambda$-systems. Hall systems and the new class of (C)-systems obtained from the exceptional (irregular) nearfields [3] are VW systems which are not $\lambda$ systems. The main aim of this paper is to deduce a set of necessary and sufficient conditions under which an arbitrary VW system is a $\lambda$-system. Using this characterization it is shown in $\$ 3$ that an isotopic or an anti-isotopic image of a $\lambda$-system is a $\lambda$-system.

2. Throughout this paper Foulser's notation [1] is followed. Let $F(+, \cdot)$ be a left VW system of order $q^{d}$ where $q=p^{s}, p$ is a prime, $d$ and $s$ are natural numbers. Let $\sigma$ be a prime such that $\sigma$ divides $\left(p^{s d}-1\right)$ but does not divide $\left(p^{s t}-1\right)$ for $0<t<d$. Prime $\sigma$ exists except in the following cases (Foulser [1, Lemma 1.1, p. 380]):

(i) $d=2, q$ is a prime of the form $2^{x}-1$;

(ii) $d=6$ and $q=2$.

Definition 2.1. Let $\tau=\sigma$ in the nonexceptional case and $\tau=2^{x}$ in the exceptional case (i).

Exceptional case (ii) does not enter our discussion since Foulser [1] proved that there are no $\lambda$-systems of order $2^{6}$ with kern $K=G F(2)$. Let $N_{l}, N_{m}$ and $K$ denote left nucleus, middle nucleus and kern in the VW system $F(+, \cdot)$ respectively.

Lemma 2.1. Let $F(+, \cdot)$ be an arbitrary (left) VW system of order $q^{2}$ where $q$ is a prime of the form $2^{x}-1$ with kern $K=G F(q)$. If $N_{l} \cap N_{m}$ contains a subgroup $G=\langle g\rangle$ of order $2^{x}$, then $F(+, \cdot)$ is generated by $\{g, 1\}$ as a right vector space over the kern $K$ where 1 is the multiplicative identity in $F(+, \cdot)$.

Proof. Since $F(+, \cdot)$ is a right vector space of dimension two over the kern $K$, the lemma is proved if it is shown that 1 and $g$ are linearly independent over $K$. Suppose there exist $a$ and $b$ in $K=G F(q)$ such that $a+g \cdot b=0$ and at least one of $a$ and $b$ are distinct from 0 . We then obtain that both $a$ and $b$ are distinct from 0 and $g=(-a) \cdot b^{-1}$ $\in G F(q)$, a contradiction since $g$ is of order $2^{x}$ and no element of $G F(q)$ is of order $2^{x}$.

Received by the editors June 16, 1969. 
Lemma 2.2. Let $F(+, \cdot)$ be a (left) Veblen Wedderburn system of order $q^{d}$ with kern $K$ of order $q=p^{*}$ where $p$ is a prime, $s$ and $d$ are natural numbers, $d>2$ and if $p=2$ and $s=1$ then $d \neq 6$. If the loop $F^{\prime}(\cdot)$ contains a power associative element $g$ of order $\tau$, then the subgroup $G=\langle g\rangle$ generates $F(+, \cdot)$ as a right vector space over $K$. Further the set $T=\left\{1, g, \cdots, g^{d-1}\right\}$ is a basis.

Proof [2, Theorem 2.1].

We now assume the following hypothesis.

Hypothesis 2.1. $F(+, \cdot)$ is a (left) VW system of order $q^{d}$ with kern $K=G F(q)$ and $q \neq 2$ if $d=6$. The group $N_{l} \cap N_{m}$ contains a cyclic subgroup $G=\langle g\rangle$ of order $\tau$ with the property $x \cdot g=g^{t(x)} \cdot x$ for all $x \in F^{\prime}$ where $t(x) \equiv q^{\mu(x)}(\bmod \sigma)$ for some mapping $\mu: F^{\prime} \rightarrow I_{d}$ (integers modulo $d$ ).

Using the fact that $g \in N_{l} \cap N_{m}$ and $g$ is of order $\tau$ the property stated in Hypothesis 2.1 may be written as

$$
x \cdot g^{k}=g^{k^{\mu}(x)} \cdot x \text { for all } x \in F^{\prime} .
$$

The following is a consequence of Lemmas 2.1 and 2.2.

Lemma 2.3. Let $F(+, \cdot)$ be a VW system satisfying Hypothesis 2.1 . Then $F(+, \cdot)$ is generated by $\left\{1, g, \cdots, g^{d-1}\right\}$ as a right vector space over the kern $K=G F(q)$.

Let $F(+, \cdot)$ be a VW system satisfying Hypothesis 2.1. Lemma 2.3 implies that if $x, y$ are arbitrary elements from $F(+, \cdot)$, then there exist elements $a_{i}, b_{i}$ in $G F(q), 0 \leqq i<d$, such that $x=\sum_{i=0}^{d-1} g^{i} \cdot a_{i}$ and $y=\sum_{i=0}^{d-1} g^{i} \cdot b_{i}$. We now define a new multiplication ' $*$ ' as

$$
x * y=\sum_{i=0}^{d-1} \sum_{j=0}^{d-1} g^{i+j} \cdot\left(a_{i} \cdot b_{j}\right) .
$$

Lemma 2.4. Let $F(+, \cdot)$ be a VW system satisfying Hypothesis 2.1. Then $F(+, *)$ is a field.

Proof. Obviously $F(+, *)$ is a commutative ring with multiplicative unity. The unity of $F^{\prime}(\cdot)$ is the unity of $F^{\prime}(*)$. Let $0 \neq x \in F$. We now show that there is a unique $y \in F^{\prime}$ such that $x * y=1$. Since $0 \neq x$, there is a unique $z \in F^{\prime}$ such that $x \cdot z=1$. Let $z=\sum_{i=0}^{d-1} g^{i} \cdot a_{i}$, $x=\sum_{i=0}^{d-1} g^{i} \cdot b_{i}$ and $y=\sum_{i=0}^{d-1} g^{i q^{\mu(x)}} \cdot a_{i}$. Then

$$
\begin{aligned}
1 & =x \cdot z=x \cdot \sum_{i=0}^{d-1} g^{i} \cdot a_{i}=\sum_{i=0}^{d-1}\left(g^{i q^{\mu(x)}} \cdot x\right) \cdot a_{i}=\sum_{i=0}^{d-1}\left(g^{i q^{\mu}(x)} \cdot \sum_{j=0}^{d-1} g^{j} \cdot b_{j}\right) \cdot a_{i} \\
& =\sum_{i=0}^{d-1} \sum_{j=0}^{d-1} g^{i q^{\mu(x)}+j} \cdot\left(a_{i} \cdot b_{j}\right)=y * x=x * y .
\end{aligned}
$$


This completes the proof of the lemma.

For any $x \in F$ let $x^{r^{*}}$ be defined inductively as $x * x=x^{2^{*}}, x * x^{(r-1)^{*}}$ $=x^{* *}$. The following are easy consequences of the definition of the multiplication $*$.

(i) $g^{r^{*}}=g^{r}$,

(ii) $g^{r} \cdot a=g^{r^{*}} * a$,

(iii) If $x=\sum_{i=0}^{d-1} g^{i} \cdot a_{i}$, then $x^{q^{* *}}=\sum_{i=0}^{d-1} g^{i q^{r}} \cdot a_{i}$

where $\langle g\rangle=G$ of Hypothesis 2.1, $a, a_{i} \in G F(q), 0 \leqq i<d$ and $x \in F$.

Lemma 2.5. A VW system $F(+, \cdot)$ satisfying Hypothesis 2.1 is a $\lambda$-system.

Proof. Let $x \neq 0 \neq y$ be arbitrary elements of $F$ with $x=\sum_{i=0}^{d-1} g^{i} \cdot a_{i}$ and $y=\sum_{i=0}^{d-1} g^{i} \cdot b_{i}$. Then

$$
\begin{aligned}
x \cdot y & =x \cdot \sum_{i=0}^{d-1} g^{i} \cdot b_{i}=\sum_{i=0}^{d-1}\left(g^{i q^{\mu(x)}} \cdot x\right) \cdot b_{i}=\sum_{i=0}^{d-1} \sum_{j=0}^{d-1} g^{i+j q^{\mu}(x)} \cdot\left(a_{i} \cdot l_{j}\right) \\
& =x * y^{q^{\mu(x)^{*}}} .
\end{aligned}
$$

(Here we have used Equations (2.1) and (2.2).) The theorem now follows from Lemma 2.1 of Foulser [1] by taking $\mu(x)$ as the mapping $\lambda(x)$ from $F^{\prime} \rightarrow I_{d}$ (integers modulo $d$ ).

Lemma 2.6. Let $F(+, 0)$ be a $\lambda$-system of order $q^{d}$ with kern $K=G F(q)$ of order $q=p^{s}$, where $p$ is a prime and $d$ and $s$ are natural numbers. Then the group $N_{l} \cap N_{m}$ contains a subgroup $G=\langle g\rangle$ of order $\tau$. If $q^{d} \neq 9$, the cyclic subgroup $G$ is the unique subgroup of order $\tau$ contained in $N_{l} \cap N_{m}$. If $q^{d}=9$, then there are three cyclic subgroups of order $\tau$ contained in $N_{l} \cap N_{m}$. Further $x \circ g=g^{a^{\lambda(x)}} \circ x$ for all $x \in F^{\prime}$ where $\lambda(x)$ is the mapping used to define the $\lambda$-system.

Proof. Let $N_{u}$ and $N_{v}$ be the subgroups of $N_{l} \cap N_{m}$ defined in $[1, \S 2.4]$. Let $\tau=2^{x}$. Then $u=(q-1)$ and $t=q+1=2^{x}=\tau$ and $N_{u}$ itself is of order $2^{x}$. In the nonexceptional case $\tau=\sigma$, the following congruences

$$
\begin{aligned}
u & \equiv 0\left(\bmod \left(q^{d}-1\right)\right) \quad \text { for } 0<m<d \text { and } m \mid d, \\
q^{d} & \equiv 1(\bmod \sigma) \text { and } q^{k} \neq \equiv 1(\bmod \sigma) \text { for } 0<k<d
\end{aligned}
$$

imply $\left(\left(q^{d}-1\right) / u\right)=t \equiv 0(\bmod \sigma)$. From this congruence it follows that $N_{u}$ contains a unique cyclic subgroup of order $\sigma$. Since $N_{u} \subseteq N_{v}$ and $N_{v}$ is a cyclic subgroup (Foulser $[1, \S 2.4]$ ), we may conclude that 
$N_{v}$ contains a unique cyclic subgroup of order $\sigma$. Let $H$ be a subgroup of $N_{l}$ of order $\sigma$ and $H=\langle h\rangle$. Since $H$ is of prime order, it is generated by every nonidentity element of $H$. Let $\lambda(h)=k$. Here $\lambda(x)$ is the mapping used by Foulser to define the $\lambda$-system. Then $\lambda(x \circ y) \equiv \lambda(x)$ $+\lambda(y)(\bmod d)$ for all $x \in N_{l}$ and all $y \in F^{\prime}($ Foulser [1, §5.1]). From this it follows that $\lambda\left(h^{d}\right) \equiv d \lambda(h)=d k(\bmod d)$ implying $\lambda(h)=0$. Since $\sigma>d, h^{d}$ is not the identity and therefore $\left\langle h^{d}\right\rangle=H$. $H$ is a subgroup of $N_{v}$ since $h \in N_{l}$ and $\lambda(h)=0$ (Foulser $[1, \S 5.1]$ ). Thus in either case $N_{l}$ contains a cyclic subgroup of order $\sigma$ which is the unique subgroup of order $\sigma$ contained in $N_{v}$. Foulser [1, Lemma 5.2, p. 387] has shown that $N_{v}$ is the unique subgroup of order $N_{v}$ contained in $N_{l} \cap N_{m}$ except in the case $q^{d}=9$ and $N_{l} \cap N_{m}$ contains three cyclic subgroups of order $\tau$ in the case $q^{d}=9$. The last part of the Lemma is a direct consequence of the definition of a $\lambda$-system.

Collecting the results of Lemmas 2.5 and 2.6 we may state the following

THEOREM 2.1. An arbitrary $V W$ system $F(+, \cdot)$ of order $q^{d}$ with kern $K=G F(q)$ of order $q=p^{s}$ where $p$ is a prime, $d$ and $s$ are natural numbers $(q \neq 2$, if $d=6)$ is a $\lambda$-system if, and only if, the group $N_{l} \cap N_{m}$ contains a cyclic subgroup $G=\langle g\rangle$ of order $\tau$ with the property $x \cdot g$ $=g^{t(x)} \cdot x$ for all $x \in F^{\prime}$, where $t(x) \equiv q^{\mu(x)}(\bmod \tau)$ for some mapping $\mu: F^{\prime} \rightarrow I_{d}$ (integers modulo $d$ ). If $q^{d} \neq 3^{2}$, the subgroup $G$ is the unique cyclic subgroup of order $\tau$ contained in $N_{l} \cap N_{m}$.

3. Let $F(+, \cdot)$ and $F_{1}(+, 0)$ be two VW systems. Let $R$ be a 1-1 additive mapping from $F$ onto $F_{1}$, and $a, b \in F^{\prime}$. If $(x \cdot y) R=(x \cdot a) R$ $\circ(b \cdot y) R$ for all $x, y \in F$, then $(R, a, b)$ is said to be an isotopism of $F(+, \cdot)$ onto $F_{1}(+, \circ)$. If $\hat{x} R \circ(x \cdot y) R=(b \cdot y) R$ for all $x, y \in F^{\prime}$, where $x \cdot \hat{x}=b \cdot a$, then $(R, a, b)$ is said to be an anti-isotopism from $F(+, \cdot)$ onto $F_{1}(+, \circ)$. The proof of the following lemma may be found in Foulser [1].

Lemma 3.1. Let $(R, a, b)$ be an isotopism (or anti-isotopism) from $F(+, \cdot)$ onto $F(+, \circ)$. Let $N_{l}$ and $N_{m}$ be left and middle nuclei respectively of $F(+, \cdot), N_{1 l}$ and $N_{1 m}$ be left and middle nuclei respectively of $F_{1}(+, \circ)$. Then $(R, a, b)$ induces the following isomorphisms:

(i) $\sigma_{l}: x \rightarrow(x \cdot b \cdot a) R$ for all $x \in N_{l}, \sigma_{l}$ is an isomorphism from $N_{l}$ onto $N_{1 l}$ (or $\left.N_{1 m}\right)$,

(ii) $\sigma_{m}: x \rightarrow(b \cdot x \cdot a) R$ for all $x \in N_{m}, \sigma_{m}$ is an isomorphism from $N_{m}$ onto $N_{1 m}$ (or $N_{1 l}$ ).

In what follows, let $F(+, \cdot)$ be a $\lambda$-system of order $q^{d}$ with kern $G F(q)$ and $F_{1}(+, 0)$ be an isotope (or an anti-isotope) of $F(+, \cdot$ ) under $(R, a, b)$. 
Lemma 3.2. The mapping $\sigma_{l}$ maps $N_{l} \cap N_{m}$ onto $N_{1 l} \cap N_{1 m}$ isomorphically.

Proof. Let $x \in N_{l} \cap N_{m}$. Then $x \sigma \in N_{1 l(m)}$ by Lemma 3.1. It may be easily verified that $x \cdot b=b \cdot x^{t}$ where $t \equiv q^{d-\lambda(b)}(\bmod \tau)$ and $x^{t} \in N_{m}$ $\cdot x \sigma_{l}=(x \cdot b \cdot a) R=\left(b \cdot x^{t} \cdot a\right) R=x^{t} \sigma_{m} \in N_{1 m(l)}$ by Lenima 3.1. It then follows that $x \sigma_{l} \in N_{1 l} \cap N_{1 m}$. Obviously $\sigma_{l}$ is an isomorphism from $N_{l} \cap N_{m}$ onto $N_{1 l} \cap N_{1 m}$.

Lemma 3.3. $N_{1 l} \cap N_{1 m}$ contains a cyclic subgroup $G_{1}$ of order $\tau$.

Proof. $N_{l} \cap N_{m}$ contains a cyclic subgroup $G$ of order $\tau$ by Theorem 2.1. It follows from Lemma 3.2 that $G \sigma_{l}$ is a desired cyclic subgroup of $N_{1 l} \cap N_{1 m}$.

Lemma 3.4. Let $(R, a, b)$ be an isotopism and $z \in F^{\prime}, x \in N_{l} \cap N_{m}$. Then $((b \cdot z) \cdot a) R \circ(x \cdot b \cdot a) R=\left(b \cdot x^{t} \cdot a\right) R \circ((b \cdot z) \cdot a) R$ where $t \equiv q^{t_{1}}(\bmod \tau)$, with $t_{1}=\lambda(b \cdot z)-\lambda(b)$.

Proof.

$$
\begin{aligned}
((b \cdot z) \cdot x) R & =((b \cdot z) \cdot a) R \circ(b \cdot x) R \\
& =((b \cdot z) \cdot a) R \circ((b \cdot x \cdot a) R) \circ(b) R .
\end{aligned}
$$

A simple computation gives $(b \cdot z) \cdot x=x^{m} \cdot(b \cdot z)=\left(x^{m} \cdot b\right) \cdot z=b \cdot x^{l} \cdot z$ where $m=q^{\lambda(b \cdot z)}, t \equiv q^{t_{1}}(\bmod \tau)$ with $t_{1}=\lambda(b \cdot z)-\lambda(b)(\bmod d)$. We then have

$$
\begin{aligned}
((b \cdot z) \cdot x) R & =\left(b \cdot x^{t} \cdot z\right) R=\left(b \cdot x^{t} \cdot a\right) R \circ(b \cdot z) R \\
& =\left(\left(b \cdot x^{t} \cdot a\right) R \circ((b \cdot z) \cdot a) R\right) \circ(b) R .
\end{aligned}
$$

From (3.1) and (3.2), it follows that

$$
((b \cdot z) \cdot a) R \circ(x \cdot b \cdot a) R=\left(b \cdot x^{t} \cdot a\right) R \circ((b \cdot z) \cdot a) R .
$$

LemMA 3.5. Let $(R, a, b)$ be an isotopism and $y \in N_{1 l} \cap N_{1 m}$ and $u \in F^{\prime}$. Then $u \circ y=y^{l} \circ u$ with $l \equiv q^{\mu(u)}(\bmod \tau)$ where $\mu(u)$ is a mapping from $F^{\prime}$ into $I_{d}$.

PROOF. $\left(b \cdot x^{t} \cdot a\right) R=\left(x^{t} \cdot q^{\lambda(b)} \cdot b \cdot a\right) R=((x \cdot b \cdot a) R)^{m}$ where $m=t \cdot q^{\lambda(b)}$ $=q^{t_{1}+\lambda(b)}=q^{\lambda(b \cdot \varepsilon)}$ since $\sigma_{l}$ is an isomorphism. Let $u=((b \cdot z) \cdot a) R$, $(x \cdot b \cdot a) R=y$. From Lemma 3.4 we obtain

$$
u \circ y=y^{l} \circ u \text { where } l=q^{\lambda(b \cdot z)}=q^{\mu(u)} .
$$

Since the mapping $R$ is $1-1$ and onto, by letting $z$ range over $F^{\prime}$ and $x$ range over $N_{l} \cap N_{m}$, we obtain that (3.3) is true for arbitrary $u \in F^{\prime}$ and arbitrary $y \in N_{1 l} \cap N_{1 m}$. Hence the lemma. 
Theorem 3.1. An isotopic image of a $\lambda$-system is a $\lambda$-system.

Proof. Let $F(+, \cdot)$ be a $\lambda$-system and $F_{1}(+, 0)$ is an isotopic image of $F(+, \cdot)$ under $(R, a, b)$. From Lemmas 3.3 and 3.5, it follows that the group $N_{1 l} \cap N_{1 m}$ contains a cyclic subgroup $G_{1}$ of order $\tau$ satisfying conditions of Theorem 2.1. Hence the theorem.

Lemma 3.6. Let $(R, a, b)$ be an anti-isotopism and $z \in F^{\prime}, x \in N_{l}$ $\cap N_{m}$. Then $((b \cdot z) \cdot a) R \circ(x \cdot b \cdot a) R=\left(b \cdot x^{t} \cdot a\right) R \circ((b \cdot z) \cdot a) R$, where $t \equiv q^{t_{1}}(\bmod \tau)$ with $t_{1}=-\lambda(u)-\lambda(b)(\bmod \alpha)$ where $u$ is the solution of the equation $u \cdot((b \cdot u) \cdot a)=b \cdot a$.

Proof. Since $(R, a, b)$ is an anti-isotopism we have

$$
\hat{x} R \circ(x \cdot y) R=(b \cdot y) R \text { for all } x, y \in F^{\prime} \text { where } x \cdot \hat{x}=b \cdot a \text {. }
$$

From (3.4) and the relations $a=((b \cdot z) \cdot a), u \cdot v=x \cdot b \cdot a$, and $u \cdot a=b \cdot a$ we obtain

$$
((b \cdot z) \cdot a) R \circ(x \cdot b \cdot a) R=\left(b \cdot x^{q^{d-\lambda(u)}} \cdot((b \cdot z) \cdot a)\right) R
$$

where $u \cdot((b \cdot z) \cdot a)=b \cdot a$. Similarly $(4.15)$ and the relations $\hat{w}=b \cdot x^{t} \cdot a$, $w \cdot e=(b \cdot z) \cdot a$, and $w \cdot \hat{w}=b \cdot a$ imply

$$
\left(b \cdot x^{t} \cdot a\right) R \circ((b \cdot z) \cdot a) R=\left(b \cdot x^{q^{d-\lambda(u)}} \cdot((z \cdot b) \cdot a)\right) R
$$

where $u \cdot((z \cdot b) \cdot a)=b \cdot a$. The lemma follows from (3.5) and (3.6).

Lemma 3.7. Let $(R, a, b)$ be an anti-isotopism and $y \in N_{1 l} \cap N_{1 m}$ and $w \in F_{1}^{\prime}$. Then wo $y=y^{l} \circ w$ with $l \equiv q^{\mu(w)}(\bmod \tau)$, where $\mu(w)$ is a mapping from $F_{1}^{\prime}$ into $I_{d}$.

Proof: Since $\sigma_{l}$ is an isomorphism from $N_{l}$ onto $N_{m}$, it follows that $\left(b \cdot x^{t} \cdot a\right) R=\left(x^{t} \cdot q^{\lambda(b)} \cdot b \cdot a\right) R=((x \cdot b \cdot a) R)^{m}$, where $m \equiv t \cdot q^{\lambda(b)}(\bmod \tau)$. Let $w=((b \cdot z) \cdot a) R$ and $y=(x \cdot b \cdot a) R$ where $z \in F^{\prime}$ and $x \in N_{l} \cap N_{m}$. Then from Lemma 3.6 we obtain

$$
w \circ y=y^{l} \circ w \text { where } l \equiv q^{d-\lambda(u)}=q^{\mu(w)} .
$$

Since the mapping is 1-1 onto, by letting $z$ range over $F^{\prime}$ and $x$ over $N_{l} \cap N_{m}$, we obtain that (3.7) is true for arbitrary $u \in F^{\prime}$ and arbitrary $y \in N_{1 l} \cap N_{1 m}$. Hence the lemma.

Theorem 3.2. An anti-isotopic image of a $\lambda$-system is a $\lambda$-system.

The proof follows from Lemmas 3.3 and 3.7 and Theorem 2.1. 


\section{REFERENCES}

1. D. A. Foulser, A generalization of André's systems, Math. Z. 100 (1967), 380395. MR 37 \#3436.

2. M. L. Narayana Rao, A question on finite Moufang Veblen Wedderburn systems, J. Algebra 13 (1969).

3. M. L. Narayana Rao, D. J. Rodabaugh, F. W. Wilke and J. L. Zemmer, A new class of finite translation planes obtained from the exceptional near-fields, J. Combinatorial Theory (to appear).

UNIVERSITY OF MisSOURI 